\title{
Facilitation of Smart car Assistant System Using IoT
}

\author{
M.Nithya ${ }^{\mathrm{a}, 1}$, M.V.S.SoundaryaLahari ${ }^{\mathrm{b}}$, J.C.E Sai Sumitra ${ }^{\mathrm{b}}$ and V. Lalitha ${ }^{\mathrm{b}}$ \\ ${ }^{a, 1}$ Department of CSE, Sri Sairam Engineering College, Chennai, India \\ ${ }^{\mathrm{b}}$ Department of CSE, Sri Sairam Engineering College, Chennai, India
}

\begin{abstract}
Human protection in cars has been the primary area of study in the past few years. A significant issue for groups of researchers and industrialists around the world is -crash- .Car users often overcome problems related to trapping in a car of car causing suffocation kind of problems, where immediate communication is very much essential. Similarly a problem often arises when it comes to the collision of vehicles from back side. This paper proposes a smart car personal assistant system that provides an efficient solution for the problems like collision detection, being trapped inside a car and also provides a necessary support by using IoT and Creation of an android application consisting of critical characteristics such as contact in the above potential problems, renewal of PUC, periodic expenditure of vehicle-related items, regular check-ups and maintenance, car database and user information of the car, which will minimize user efforts.
\end{abstract}

Key-words: Suffocation, Collision, Smart Car, Arduino, Alerts, Monitoring.

\section{Introduction}

\subsection{Domain Overview}

The Internet of Things (IoT) is a subtle collection of technologies and uses a simple, single concept of inbound instances .One effective framework for viewing IoT such as the use of network-connected devices, entrenched in a visible environment, to enhance the present process. These devices, or objects, are connected to a network to supply information which would be collected from an environment with the help of the sensors. They can be connected by phone to common objects that you may already be familiar with, or to new devices designed for the purpose of unreachable activities. The IoT environment is only beginning with enormous use cases and possibilities through diverse industries and scenarios that set a common challenge and pattern.

\subsection{Objective}

The key objective of this paper is to provide a secured and an efficient solution for the consistent and evident problems in daily life. Considering the human safety as a major 
factor in non-crash related accidents in car, the proposed system is capable of-Sensing the suffocation levels of at rapped occupant, Sensing the presence of human in a turned off car, Intimating the people around and users connected about the fatal situations Alerting users by detecting cars to prevent rear-end collisions. The most essential part considering the safety and comfort in automobile is 'automation of anti-suffocation system'. The big concern is the death in locked vehicle soft toddlers, disabled person sand animal sowing to Asphyxiation. Therefore, a device that automatically works with minimum power consumption and disbursement is required to address this form of threat to human life. Heat stroke in automobiles is the leading cause of most crashrelated fatalities, Pursuant to the National Highway Traffic Safety Administration (NHTSA). 556 children in the United States of America have died in a locked car since 1998. (USA). Children under the age of 15 accounted for nearly 15 per cent of trafficfree deaths between 2006 and 2010. One child dies in the U.S. every 10days due to a vehicle heatstroke. An average of 53 children die in hot cars each year after being trapped in motor vehicles due to suffocation. It can take up to 15 minutes for kids to suffer from malignant brain-kidney injuries in a heated vehicle. [The Hindu June 13,2015]In a recent case, two kids has been trapped in this situation where they took the car keys to play inside. When they closed the door, the car was centrally locked .Sadly, the two sisters didn't know how to unlock the door, and the windows were closed and locked. Parents were unaware of it and checked inside and outside the home. They found the missing car key several hours later and eventually, they found their kids dead inside. They emanated to realize later that suffocation was the cause of death. The main reason behind these fatalities is that when vehicles are parked without overhead garages in a hot environment and with the engine off, doors and windows locked and outside temperatures above $33^{\circ} \mathrm{C}$, the inside temperature will rise in a short time above the comfort limit. This may hyperthermia for a child or disabled. It clearly describes the state of observation that most percentage of people end up leaving their children in the cars for shopping which they think would be a short time work .But at eventual delay of shopping coincides with the accidental lock of car then this would cause an unexpected accident which could result anything. This pie chart does not includes the cases where physically challenged people may also end up locked inside car due to some reasons. This is because there is no such accidents caused and the prediction of happening this is $0.089 \%$ according to researchers. With the rise in deaths due to the suffocation epidemic, vehicle surveillance and child protection/human safety inside a car has become a considerable problem all over the world. There are no existing and systematic solutions in the scenario for law enforcement agencies (LEAs) and vehicle tracking for the safety of the child / person inside the car. Our endowment is to provide reasonable proof of the prototype. To track the child inside the car and provide a security solution with instant data control feature, we provide an advanced solution framework. Below is the main goal of the work of the research project, which will lead us to improve the prototype further. The presence of adults, children and luggage in a car seat should be separated, To have the controlling intervention to save a child's life. When it comes to the crash related accidents, Road accidents are the most unwanted things to happen. The general rules and safety measures are well known to most road users when using highways, but it is really the sensitivity of road users that causes accidents and crashes. The main cause of accidents and crashes is human error. One those errors is the distraction. The distraction may be outside or inside the vehicle. 
Talking on a mobile phone while driving is now a big distraction. A large part of the brain is consumed by the activity of talking on the phone and a small part retains driving skills. This brain division impairs reaction time and the ability to judge. This can because for crashes. Attending the telephone calls while driving should be avoided. You must pull out on the side of the road to take part in the call if the call is urgent .Adjusting mirrors, vehicle stereo/radio, road animals ,banners and billboards while driving on the road are necessary. These distractions can cause the collision between vehicles . To avoid those type of collisions, the driver must be alerted every time to avoid collision of vehicles. The present system provides frequent monitoring of the vehicles coming from backside and alerts the user with visual and audio interactions.

\section{Literature Survey and System Analysis}

In the recent years many researchers focus on IoT based application. Jetendra Joshi\& co [1] focused on functioning of the sensors and their correlation with alert system. Angelamartin et al [2] discussed about the calculation of suffocation level. Smit Grethiya Himanshu Agarwal\& co [3] discussed the connection of the android components with the sensors. Neelam Sharma[4]discussed about the basic functionality of the $\mathrm{CO} 2$ sensor and its working. The already existing system finds the solution of this problem by detecting the $\mathrm{Co} 2$ level up to the suffocating mark using gas sensors. After the calculation of the $\mathrm{Co} 2$ level, once after it reaches the peak level, the mechatronics are implied. The windows are automatically glided down. Though, this method proves to be effective, it fails when considering car safety without human intervention. The glass cabin is used here to achieve a volume constant of $30 \times 30 \times 30$ cubic.cm. In the top of the glass tank, two holes are made where one for $\mathrm{CO} 2$ sensor and the other one for $\mathrm{CO} 2$ gas supply. The sensor senses the preset threshold level of $\mathrm{CO} 2$ when air from the $\mathrm{CO} 2$ tank exits the glass tank, and a digital signal is sent to the Arduino UNO PIN 8. Using the Arduino UNO compiler on the Machine Interface to programs the Arduino UNO uploads via USB. The Arduino board sends the output digital signal to the L298Ndual motor controller module - 2A after receiving the input signal from the sensor. DC motor is been controlled by a power window which establishes a connection between the When the motor runs, the power window goes down and allows fresh air. The following components are attached to the breadboard Arduino board,L298Ndual motor controller module-2A,DC motor ,CO2sensor. The permissible atmospheric $\mathrm{CO} 2$ levels for human survival and the estimation of the $\mathrm{CO} 2$ PPM level based on the chosen test space are determined by the following equation $\%$ Concentration $=\mathrm{ppm} / 10,00$.This existing system has some draw backs that has to be concentrated and could be used for reference of a new system.1.Lowering the window each and every time $\mathrm{CO} 2$ decrease may seriously lead to potential security threat and issues.2.Similarly, the permission from the particular user is not needed at this type of system. So, this may lead to genuineness problem. Even any type of gas in the car at high proportions may directly head to opening of windows which is inaccurate.

\section{Proposed System}

The proposed system provides the features of solving two main problems caused by the crash and non-crash related accidents. This type of system is one of the fastest growing 
safety features in the automotive industry. Such a system allows vehicles to detect the possibility of collisions and to issue visual and audio alerts to the driver so that the driver can take the necessary steps to avoid collision. It also provides an efficient suffocation detection which can prevent the danger rates caused due to asphyxiation and manages to alert the user and surroundings. The implementation details are as follows:The project is combined with Arduino components, sensors and the alert system. The Arduino controller is connected with the two main sensors which plays the key role of the project. The CO2 Sensor (MQ135Gassensor) and the distance calculation sensor (Ultra sonic distance sensor) are connected with Arduino UNO. The threshold level of $\mathrm{CO} 2$ are kept as benchmark considering the information.

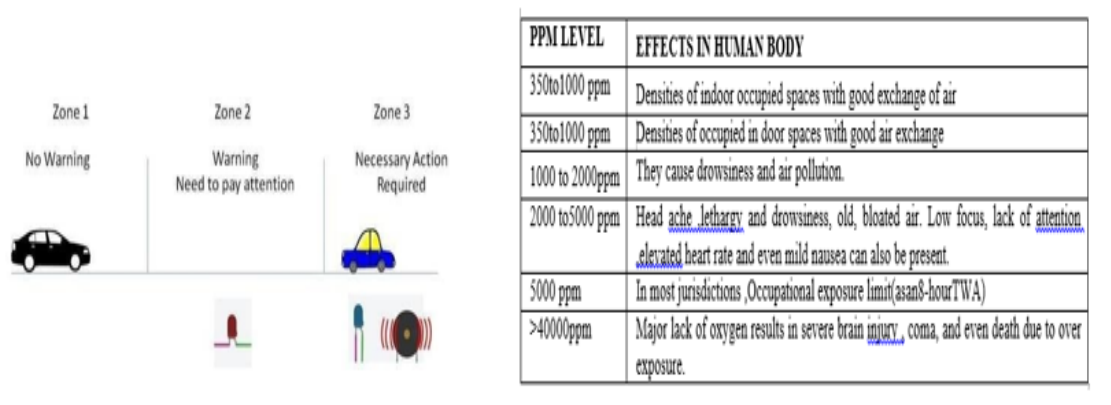

Figure 1. Working of Collision Management

The parts assembly as shown in the Arduino Uno is connected with the Co2 gas sensor and the ultrasonic sensor .Breadboard connects all the components on it acting as a base and power distributor. The car on and off are stated with the help of the switch. When the switch is off, the output from the CO2 sensor is verified, if the level reaches its peak the control is transferred to the buzzer .Similarly, when the switch is ON which states that the car is in working state, then it continuously detects the collision of objects from distance. Once the distance crosses the level, the driver is indicated with visual and audible interactions with the help of LED and buzzer. As the distance calculation is based on the ultrasonic sensor, it is important to know that the module has two eyes like projects in the front which forms the Ultrasonic transmitter and Receiver. The ultrasonic transmitter transmits an ultrasonic wave, this wave moves in air, and this reflected wave is detected by the Ultrasonic receiver as it gets opposed by some material it gets reflected back towards the sensor.

\section{Discussion of Result}

At a case , where the car is OFF the suffocation levels are detected very frequently and is compared with the peak level. Once it reaches, the peak level the control is taken by the alert system. At a case, where the car is ON the collision chances are detected very frequently by checking the distance with the minimum distance value. Once it crosses the minimum distance, the driver is notified with visual and audible warnings and alerts. The corresponding measurements and the outputs can be viewed by the user . 


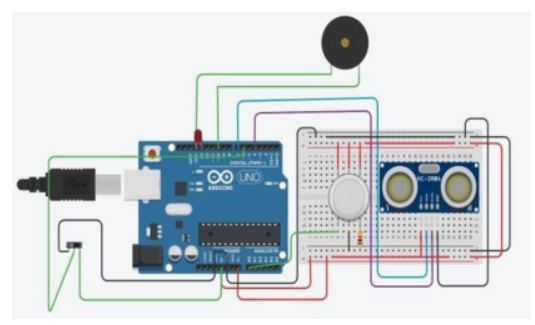

Figure 2. Circuit Diagram of Proposed System

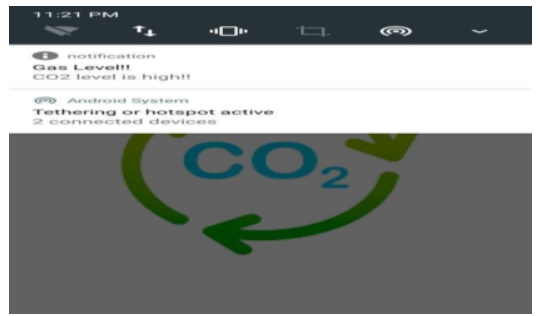

Figure 3. User notification

\section{Conclusion of the System}

The intimation of someone being locked and is suffocated in the car can be efficiently done without any regrets. In the emergency cases, user has an optional choice of sending this particular notification to the police or anyone of his choice whom he could trust. With positive test results, the In-Car Suffocating Prevention Framework can be successfully implemented as it can detect a person inside a parked car. Human lives can be saved within a 30-minute cycle as this avoidance device warns the owner to check for any defective purpose due to his or her carelessness. In order to identify humans, the device uses a very simple automation technique where feature extraction of a human presence is analyzed. By doing this, after being left alone for a certain amount of time for some reason, the device will help avoid child or pet death inside a vehicle. Similarly this could be the best efficient solution to alert the driver from all the distractions by checking the colliding objects. During such critical cases, this technique proves to be effective and easy to save lives. It enhances protection without any human intervention inside the vehicle.

\section{References}

[1] Jetendra Joshi, Vehicle and Passenger protection through cooperative sensor based Vehicular Networking , 2017 IEEE 4th International conference on smart Instrumentation Conference on Smart Instrumentation, Measurement and Application.

[2] Ambeth Kumar.V.D, G.Saranya , D.Elangovan, V.RahulChiranjeevi, V.D.Ashok Kumar.IOT Based Smart Museum Using Wearable Device.Lecture Notes in Networks and Systems, Vol.55, pp: 33-42, 2018

[3] Smit Grethiya Himanshu Agarwal,Shilpa Gite and Suresh V ,Affordable system for alerting, monitoring and controlling heat stroke inside vehicles, at Industrial Instrumentation and Control (ICIC),2015.

[4] Neelam Sharma and Anurag Tyagi, An IoT Approach for motion detection using Raspberry pi, International Conference on Intelligent Computing and Internet of Things (ICIT),2017.

[5] Kids and car, 2016, Children death due heatstroke in car. :http://www.kidsandcars.org/heatstroke.html

[6] National highway traffic safety administration. 2015, heatstroke death in car. Available from :https://www.nhtsa.gov/heatstroke

[7] Liu C. (2001).US6263272.USA: Google Patents

[8] Liu j. (2000).US6138068.USA: Google Patents 